\title{
Immunosuppression by monocytic myeloid-derived suppressor cells in patients with pancreatic ductal carcinoma is orchestrated by STAT3
}

Rosalinda Trovato ${ }^{1 \dagger}$, Alessandra Fiore ${ }^{1,2+}$, Sara Sartori ${ }^{1 \dagger}$, Stefania Canè ${ }^{1 \dagger}$, Rosalba Giugno ${ }^{3}$, Luciano Cascione $^{4}$, Salvatore Paiella ${ }^{5}$, Roberto Salvia ${ }^{5}$, Francesco De Sanctis ${ }^{1}$, Ornella Poffe ${ }^{1}$, Cristina Anselmi ${ }^{1}$, Francesca Hofer ${ }^{1}$, Silvia Sartoris ${ }^{1}$, Geny Piro ${ }^{6,7}$, Carmine Carbone ${ }^{6,7}$, Vincenzo Corbo ${ }^{8,9}$ (D), Rita Lawlor ${ }^{9}$, Samantha Solito ${ }^{10,11}$, Laura Pinton $^{10}$, Susanna Mandruzzato ${ }^{10,12}$, Claudio Bassi ${ }^{5}$, Aldo Scarpa ${ }^{8,9}$, Vincenzo Bronte ${ }^{1 *}$ and Stefano Ugel ${ }^{1}$

\begin{abstract}
Background: Pancreatic ductal adenocarcinoma (PDAC) is a highly devastating disease with an overall 5-year survival rate of less than $8 \%$. New evidence indicates that PDAC cells release pro-inflammatory metabolites that induce a marked alteration of normal hematopoiesis, favoring the expansion and accumulation of myeloid-derived suppressor cells (MDSCs). We report here that PDAC patients show increased levels of both circulating and tumor-infiltrating MDSC-like cells.
\end{abstract}

Methods: The frequency of MDSC subsets in the peripheral blood was determined by flow cytometry in three independent cohorts of PDAC patients (total analyzed patients, $n=117$ ). Frequency of circulating MDSCs was correlated with overall survival of PDAC patients. We also analyzed the frequency of tumor-infiltrating MDSC and the immune landscape in fresh biopsies. Purified myeloid cell subsets were tested in vitro for their T-cell suppressive capacity.

Results: Correlation with clinical data revealed that MDSC frequency was significantly associated with a shorter patients' overall survival and metastatic disease. However, the immunosuppressive activity of purified MDSCs was detectable only in some patients and mainly limited to the monocytic subset. A transcriptome analysis of the immunosuppressive M-MDSCs highlighted a distinct gene signature in which STAT3 was crucial for monocyte re-programming. Suppressive M-MDSCs can be characterized as circulating STAT3/arginase1-expressing CD14 ${ }^{+}$cells. Conclusion: MDSC analysis aids in defining the immune landscape of PDAC patients for a more appropriate diagnosis, stratification and treatment.

Keywords: Myeloid-derived suppressor cells (MDSC), Pancreatic ductal adenocarcinoma (PDAC), Innate immunity, Tumorassociated immunosuppression, Tumor progression

\footnotetext{
* Correspondence: vincenzo.bronte@univr.it

${ }^{\dagger}$ Rosalinda Trovato, Alessandra Fiore, Sara Sartori and Stefania Canè

contributed equally to this work.

${ }^{1}$ University Hospital and Department of Medicine, Section of Immunology,

University of Verona, Verona, Italy

Full list of author information is available at the end of the article
}

(c) The Author(s). 2019 Open Access This article is distributed under the terms of the Creative Commons Attribution 4.0 International License (http://creativecommons.org/licenses/by/4.0/), which permits unrestricted use, distribution, and reproduction in any medium, provided you give appropriate credit to the original author(s) and the source, provide a link to the Creative Commons license, and indicate if changes were made. The Creative Commons Public Domain Dedication waiver (http://creativecommons.org/publicdomain/zero/1.0/) applies to the data made available in this article, unless otherwise stated. 


\section{Background}

Over the last thirty years, pancreatic ductal adenocarcinoma (PDAC) worldwide incidence has increased significantly and PDAC ranks the fourth leading cause of cancer death with a 5-year survival time of less than 8\% [1]. Despite many new treatments, including immune modulation, pancreatic cancer remains highly resistant to therapy $[2,3]$. The presence of the highest degree of desmoplasia among all solid tumors and the occurrence of a chronic inflammation endorse a critical role for tumor microenvironment on pancreatic carcinogenesis $[4,5]$. In preclinical models, by releasing high amounts of growth factors such as granulocyte colonystimulating factor (G-CSF) and granulocyte-macrophage colony-stimulating factor (GM-CSF), pancreatic tumor cells activate an abnormal myelopoiesis that promotes the recruitment of a heterogeneous population of myeloid cells characterized by a strong immunosuppressive activity $[6,7]$. These cells are termed myeloid-derived suppressor cells (MDSCs) [8] and their accumulation, in the blood and at the tumor site, has been associated with advanced tumor stage and unfavorable prognosis in several human malignancies [9].

The main feature of MDSCs is the ability to switch off adaptive and innate immune responses [10]. Indeed, MDSCs are able to release both reactive oxygen species (ROS) and reactive nitrogen species (RNS), which inhibit $\mathrm{T}$ cells fitness, proliferation and migration within the tumor microenvironment; MDSCs deplete essential metabolites by activating key enzymes such as arginase-1 (ARG1) and indoleamine 2,3-dioxygenase 1 (IDO1), which are capable of reducing Larginine and L-tryptophan availability, respectively. In addition, MDSCs induce $\mathrm{T}$ cell tolerance through the expression of inhibitory receptors such as the programmed death-ligand 1 (PD-L1) and the cytotoxic T-lymphocyte antigen 4 (CTLA-4) receptors, as well as they sustain the development of regulatory $\mathrm{T}$ cells (Treg) through the CD40 engagement in presence of inteleukin-10 (IL-10) and transforming growth factor beta (TGF $\beta)$ [11]. All these immunosuppressive mechanisms are the result of altered signaling pathways leading to induction of transcriptional factors such as the nuclear factor kappa-light-chain-enhancer of activated B cells $(\mathrm{NF}-\mathrm{kB})$ [12], the CCAAT-enhancer-binding proteins ( $\mathrm{c} /$ EBP) $-\beta$ [13] and members of the signal transducer and activator of transcription (STAT) family, like STAT3 [14]. Besides immune regulation, MDSCs favor tumor progression also by non-immune properties, promoting tumor angiogenesis and vasculogenesis as well as cancer cell stemness, aggressiveness and invasiveness [11].

In mice, MDSCs were classically identified as $\mathrm{CD}_{11 \mathrm{~b}^{+} \mathrm{Gr}-}$ $1^{+}$cells and divided into two main subgroups: polymorphonuclear (PMN)-MDSCs (CD11 b ${ }^{+} \mathrm{Ly}_{6} \mathrm{G}^{+} \mathrm{Ly}_{6} \mathrm{C}^{\mathrm{lo}}$ cells) and

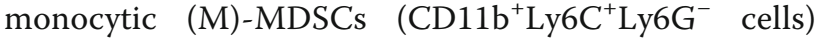
[6]. In humans instead, three major MDSC subsets have been identified: PMN-MDSCs, M-MDSCs and "early-stage MDSCs" (e-MDSC) [15]. Since human MDSCs display surface markers shared with normal myeloid cell subsets (such as CD14, CD15, and CD33) and exhibit an intrinsic heterogeneity and plasticity, it is mandatory to integrate the phenotypical characterization with functional assays demonstrating their authentic immunosuppressive functions [15].

Here we applied standardized flow cytometry methods to distinguish and enumerate circulating MDSCs in both whole blood (WB) and frozen PBMCs obtained from three independent cohorts of PDAC patients; additionally, we analyzed the frequency of tumor-infiltrating MDSC and the immune composition in freshly isolated biopsies. Finally, we tested the immunosuppressive functions of circulating, purified MDSCs by evaluating their ability to control in vitro proliferation of activated $\mathrm{T}$ cells. Since only the M-MDSC subset showed robust inhibitory properties, we further exploited their transcriptomic profile with the aim at identifying novel biomarkers and specific molecular pathways.

\section{Material and methods \\ Human samples collection}

Peripheral blood samples were prospectively collected from three independent cohorts of patients with different stages of pancreatic ductal adenocarcinoma admitted at the Unit of General and Pancreatic Surgery of the Azienda Ospedaliera Universitaria Integrata of Verona before surgical resection or Healthy Donors (HD). Clinic-pathologic features of patients were reported in Tables 1 and 2 and

Table 1 Clinical characteristics of the study population

Patients Cohort $(n=29)$

\begin{tabular}{ll}
\hline Gender & 55.2 \\
Male (\%) & 44.8 \\
Female(\%) & $66(48-85)$ \\
Age (Range) & \\
Stage & 48.3 \\
IIA-IIB (\%) & 51.7 \\
III (\%) & 0.0 \\
IV(\%) & \\
Tumor site & 86.2 \\
Head & 10.3 \\
Body & 3.5 \\
Tail & 0.0 \\
Multi localized
\end{tabular}

The total number of cases, the male/female percentage, the mean age (years) with minimum and maximum value (range), stage and tumor localization of the analyzed cohort of PDAC patients 
Table 2 Clinical characteristics of the study population

\begin{tabular}{llll}
\hline & Cohort_1 $(n=21)$ & Cohort_2 $(n=23)$ & Cohort_3 $(n=73)$ \\
\hline Gender & & & 30.4 \\
Male (\%) & 61.9 & 69.6 & 53.4 \\
Female(\%) & 38.1 & $67(47-84)$ & 46.6 \\
Age (Range) & $67(52-79)$ & & $65(40-82)$ \\
Stage & & $/$ & 28.7 \\
I-II (\%) & $/$ & 56.5 & 39.7 \\
III (\%) & 57.1 & 43.5 & 31.6 \\
IV(\%) & 42.9 & & 64.7 \\
Tumor site & & 26.1 & 19.1 \\
Head & 43.3 & 47.8 & 6.8 \\
Body & 29.0 & 17.4 & 9.4 \\
Tail & 19.0 & 8.7 & \\
Multi localized & 8.7 & & \\
\hline
\end{tabular}

The total number of cases, the male/female percentage, the mean age (years) with minimum and maximum value (range), stage and tumor localization of the three cohorts of PDAC patients. In each cohort, PDAC patients were compared to a cohort of age- and gender-matched healthy donors

included age, gender, tumor location and TNM stage. No subject had a prior history of cancer or was undergoing therapy at the time of sample collection. BM aspirates were subjected to lysis to remove red blood cells, with a hypotonic solution of ammonium chloride. Cells were plated $\left(2 \times 10^{6}\right.$ cells/well $)$ into a 24-well tissue culture plate (BD, Franklin Lakes, NJ, USA) in IMDM (Lonza, Visp, Switzerland) supplemented with with 10\% FBS (Euroclone, Milano, Italy), $100 \mathrm{U} / \mathrm{ml}$ penicillin/streptomycin (Euroclone, Milano, Italy), $\beta$-mercaptoethanol (Sigma-Aldrich, Milan, Italy) and $10 \mathrm{mM}$ HEPES (Euroclone, Milano, Italy) in presence of $40 \mathrm{ng} / \mathrm{ml}$ of G-CSF and GM-CSF (Miltenyi Biotec) for 4 days at $37^{\circ} \mathrm{C}, 8 \%$ $\mathrm{CO}_{2}$, obtaining BM-MDSC as previously reported [16].

\section{Human proliferation assay}

PBMCs were isolated from leukocyte-enriched buffy coats from healthy volunteers (Transfusion Center, University and Hospital Trust of Verona, Verona, Italy) by FicollHypaque (GE Healthcare, Uppsala, Sweden) gradient centrifugation. PBMCs were then counted, frozen at $-80^{\circ} \mathrm{C}$ and stored in liquid nitrogen. PBMCs were recovered, washed in IMDM medium (Lonza, Visp, Switzerland), supplemented with 10\% FBS (Euroclone, Milano, Italy), $100 \mathrm{U} /$ $\mathrm{ml}$ penicillin/streptomycin (Euroclone, Milano, Italy), $\beta$ mercaptoethanol (Sigma-Aldrich, Milan, Italy) and $10 \mathrm{mM}$ HEPES (Euroclone, Milano, Italy), resuspended at a final concentration of $10^{7}$ cells $/ \mathrm{ml}$ in PBS and stained with $1 \mu \mathrm{M}$ as final working concentration of CellTrace Violet stock solution (Thermo Fisher Scientific, Waltham, MA, USA), followed by $5 \mathrm{~min}$ ' incubation at $37^{\circ} \mathrm{C}$, protected from light. Labelled "target" PBMCs were stimulated with coated $0.6 \mu \mathrm{g} / \mathrm{ml}$ anti-CD3 (clone OKT-3, eBioscience, Thermo Fisher Scientific, Waltham, MA, USA) and $5 \mu \mathrm{g} /$ $\mathrm{ml}$ soluble anti-CD28 (clone CD28.2, eBioscience, Thermo Fisher Scientific, Waltham, MA, USA) for 4 days and cocultured with "effectors" M-MDSCS $\left(\mathrm{CD} 14^{+}\right.$cells) or PMN-MDSC $\left(\mathrm{CD} 6 \mathrm{~b}^{+}\right.$cells) cells at 0.5:1, 1:1, 3:1, 6:1 ratio (effector:target) in 384 flat bottom well plates (BD, Franklin Lakes, NJ, USA). Cell cultures were incubated at $37^{\circ} \mathrm{C}$ and $8 \% \mathrm{CO}_{2}$ in arginine and glutamine-Free-RPMI (Biochrom AG, Berlin, Germany), supplemented with $2 \mathrm{mML-glu-}$ tamine (Euroclone, Milano, Italy), $150 \mu \mathrm{M}$ arginine (SigmaAldrich, St. Louis, MO, USA), 10\% FBS (Superior, Merck, Darmstadt, Germany), $10 \mathrm{U} / \mathrm{ml}$ penicillin and streptomycin (Euroclone, Milano, Italy), and 0.1 mM HEPES (Euroclone, Milano, Italy). At the end of the culture, cells were stained with PE-Cy7 conjugated anti-CD3 (UCHT1, eBioscience, Thermo Fisher Scientific, Waltham, MA, USA), and CellTrace signal of gated lymphocytes was analyzed. TruCount $^{\text {ti }}$ tubes (BD, Franklin Lakes, NJ, USA) were used to determine the absolute cell number of $\mathrm{CD}^{+}$cells in the samples. Data were analyzed by FlowJo software (Tree Star, Inc. Ashland, OR, USA).

\section{Human cell preparation and flow cytometric analysis}

Blood was collected into EDTA-treated tubes (BD Biosciences, NJ, USA) and processed fresh. For each donor, $450 \mu \mathrm{L}$ of whole blood or $10^{6}$ frozen PBMCs were taken for MDSC characterization by flow cytometry. Sample tubes were washed in phosphate-buffered saline (PBS), incubated with $\mathrm{Fc}_{\mathrm{c}}$ receptor ( $\mathrm{FcR}$ ) Blocking reagent (Miltenyi Biotec) for $10 \mathrm{~min}$ at $4{ }^{\circ} \mathrm{C}$ to saturate FcR and then stained with fluorochrome-conjugated antibodies (Additional file 1: Supplementary methods). For tumorinfiltrating leukocytes evaluation, tumor biopsies were minced and incubated for $2 \mathrm{~h}$ at $37^{\circ} \mathrm{C}$ with shaking with an enzymatic cocktail. Normal tissues were detected by 
pathological analysis and isolated from patient biopsies. $5 \times 10^{5}$ cells were washed with PBS supplemented with 2 Mm EDTA, incubated with FcR Blocking reagent (Miltenyi Biotec) for $10 \mathrm{~min}$ at $4{ }^{\circ} \mathrm{C}$ and then stained with fluorochrome-conjugated antibodies (Additional file 1: Supplementary methods).

\section{RNA isolation and gene expression}

Total RNA was isolated using TRIzol reagent (Life technology, CA, USA) and RNA integrity assessed using Agilent-2100-Bioanalyzer (Agilent Technologies, CA, USA). RNA from human $\mathrm{CD}_{14}{ }^{+}$cells was further purified with RNeasy MinElute Cleanup kit (Qiagen, Venlo, Netherlands) and cDNA was synthesized and amplified from total purified RNA with RETROscript ${ }^{\circ}$ (Life technology, CA, USA). All the samples were hybridized to Affymetrix U133 PLUS 2.0 arrays and scanned with an Affymetrix GCS 3000 7G scanner.

\section{Statistical analysis}

All statistical analysis was carried out using SigmaPlot (Systat Software) and R/Bioconductor. For statistical comparison of two groups, non-parametric MannWhitney Wilcoxon test was used. Data are shown as mean \pm SD or mean \pm SEM as indicated in the figures legends. Receiver operator characteristic (ROC) analysis was performed to determine the performance of MDSC percentage in distinguishing patients with metastatic carcinoma. The optimal cutoff threshold for MDSC percentage was obtained based on the maximization of the Youden's statistics $\mathrm{J}=$ sensitivity + specificity +1 by using an R-based software as described [17]. Statistical analyses were performed using SPSS Statistics 22 (IBM Corporation, Somers, NY, USA), GraphPad Prism software program (version 6.0; GraphPad Software, San Diego, CA), and the statistical language $R$.

\section{Results}

\section{The frequency of tumor-infiltrating $T$ cells inversely} correlates with the presence of PMNs and M-MDSCs

The immune composition of PDAC has been shown to have prognostic implications, with high number of $\mathrm{CD}^{+} \mathrm{T}$ lymphocytes associated with good outcome while accumulation of myeloid cells with poor prognosis $[18,19]$. However, our knowledge on the immune heterogeneity of the PDAC microenvironment is still limited and need to be further investigated. To dissect this complexity, using a multicolour flow cytometry approach, we analysed infiltrating leukocytes isolated from 29 tumor samples from treatment-naïve PDAC patients (Table 1) and 5 normal pancreatic biopsies, obtained from tumor-free tissues of some patients. Among alive $\mathrm{CD} 45^{+}$cells, we focused on T lymphocytes $\left(\mathrm{CD}^{+}\right.$cells), effector $\mathrm{T}$ lymphocytes $\left(\mathrm{CD}^{+} \mathrm{CD}^{+}\right.$cells), helper $\mathrm{T}$ lymphocytes $\left(\mathrm{CD}^{+} \mathrm{CD} 4^{+}\right.$cells), regulatory $\mathrm{T}$ lymphocytes $\left(\mathrm{CD}^{+} \mathrm{CD}^{+} \mathrm{CD}^{+} 5^{+} \mathrm{FoxP}^{+}\right.$cells, Tregs), B lymphocytes $\left(\mathrm{CD}^{-} \mathrm{CD} 19^{+}\right.$cells), regulatory $\mathrm{B}$ cells $\left(\mathrm{CD}^{-} \mathrm{CD} 19^{+} \mathrm{CD} 25^{+} \mathrm{FoxP}^{+}\right.$cells, Bregs), myeloid-dendritic cells $\left(\mathrm{CD} 11 \mathrm{~b}^{+} \mathrm{CD} 11 \mathrm{c}^{+} \mathrm{HLA}-\mathrm{DR}^{+}\right.$cells, DCs), plasmacitoid DCs $\left(\mathrm{CD} 11 \mathrm{~b}^{+} \mathrm{CD} 11 \mathrm{c}^{-} \mathrm{CD} 123^{+}, \mathrm{pDCs}\right)$, macrophages (CD

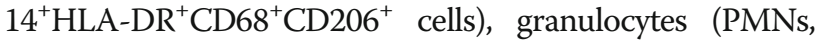
$\mathrm{CD} 14^{-} \mathrm{CD} 15^{+} \mathrm{CD} 11 \mathrm{~b}^{+}$cells) as well as two MDSC subsets: e-MDSCs ( Lin $^{-}$HLA-DR ${ }^{-}$CD $11 \mathrm{~b}^{+} \mathrm{CD} 33^{+}$cells) and M-

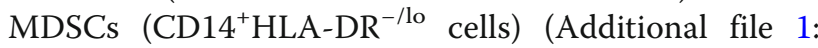
Figure S1). Notably, we found that PDAC tissues have a higher $\mathrm{CD} 45^{+}$cell infiltrate than their normal counterpart, likely reflecting the ability of the tumor or surrounding stroma to release soluble factors attracting immune cells $[20,21]$ (Fig. 1a). Among the $\mathrm{CD} 45^{+}$cells, we identified a high frequency of several myeloid cells, such as PMNs, MDSCs and macrophages (Fig. 1b) and several T cell subsets, supporting the current hypothesis that PDAC is not an immune "desert" [22, 23]. While we did not find expansion in Bregs $(0.052 \pm 0.012)$ and pDCs $(0.073 \pm$ 0.018 ), we observed a higher frequency of several myeloid cells, such as PMNs $(28.89 \pm 4.693)$, M-MDSCs $(0.969 \pm$ $0.167)$, e-MDSC (1.235 \pm 0.198$)$ and macrophages $(8.832 \pm$ $2.265)$ and Tregs $(1.092 \pm 0.196)$ (Fig. 1b), supporting the concept that PDAC is a tumor with an immune-hostile tumor microenvironment [24]. Indeed, a significant inverse correlation between $\mathrm{T}$ cell numbers with either PMN or M-MDSCs, but not with macrophages and e-MDSCs, could be detected (Fig. 1c), which is in line with recent reports. Of note, a significant inverse correlation between both PMNs and B cells, as well as between PMNs and different $\mathrm{T}$ cell subsets including effectors $\mathrm{T}$ cells, helper $\mathrm{T}$ cells and Tregs emerged (Additional file 1: Figure S2). Interestingly, we identified a significant direct linear correlation between $\mathrm{T}$ cells and Tregs as well as a trend between M-MDSCs and Tregs (Additional file 1: Figure S2). Collectively, these results suggest that accumulation of myeloid cells, such as MDSCs, in PDAC is detrimental for $\mathrm{T}$ cell infiltration.

\section{PDAC patients exhibit a significant increase in circulating MDSCs}

Preclinical data suggest that PDAC effects on the immune system are not limited to local microenvironment but might cause systemic alterations, fuelling an "emergency" myelopoiesis that favours the accumulation of circulating MDSCs [13]. To assess systemic changes in PDAC patients, we evaluated the presence of different MDSC subsets in the peripheral blood, following recently published guidelines [25]. Three independent patient cohorts (Table 2) were enrolled to define the MDSC baseline at diagnosis: the first cohort comprised 21 PDAC patients (stage III-IV) and 8 age- and sex-matched healthy donors (HD) (Fig. 2a), the second cohort comprised 23 PDAC 


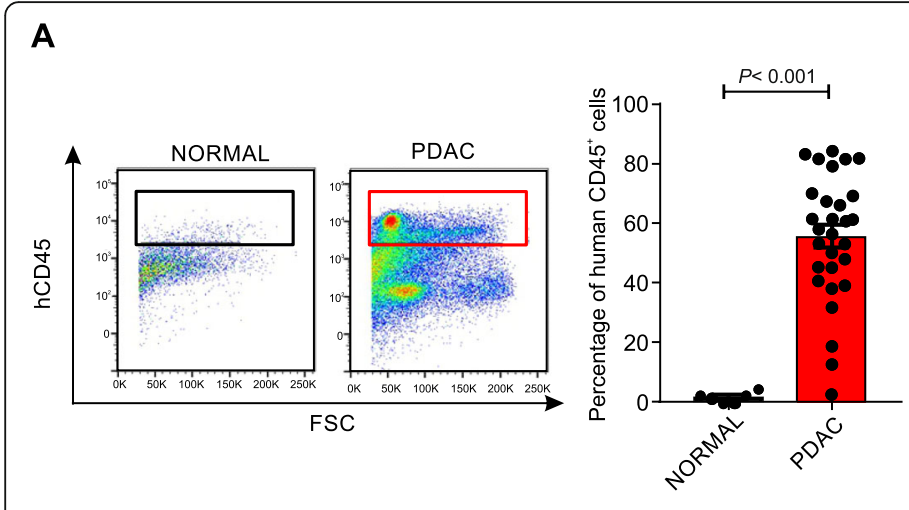

B

C
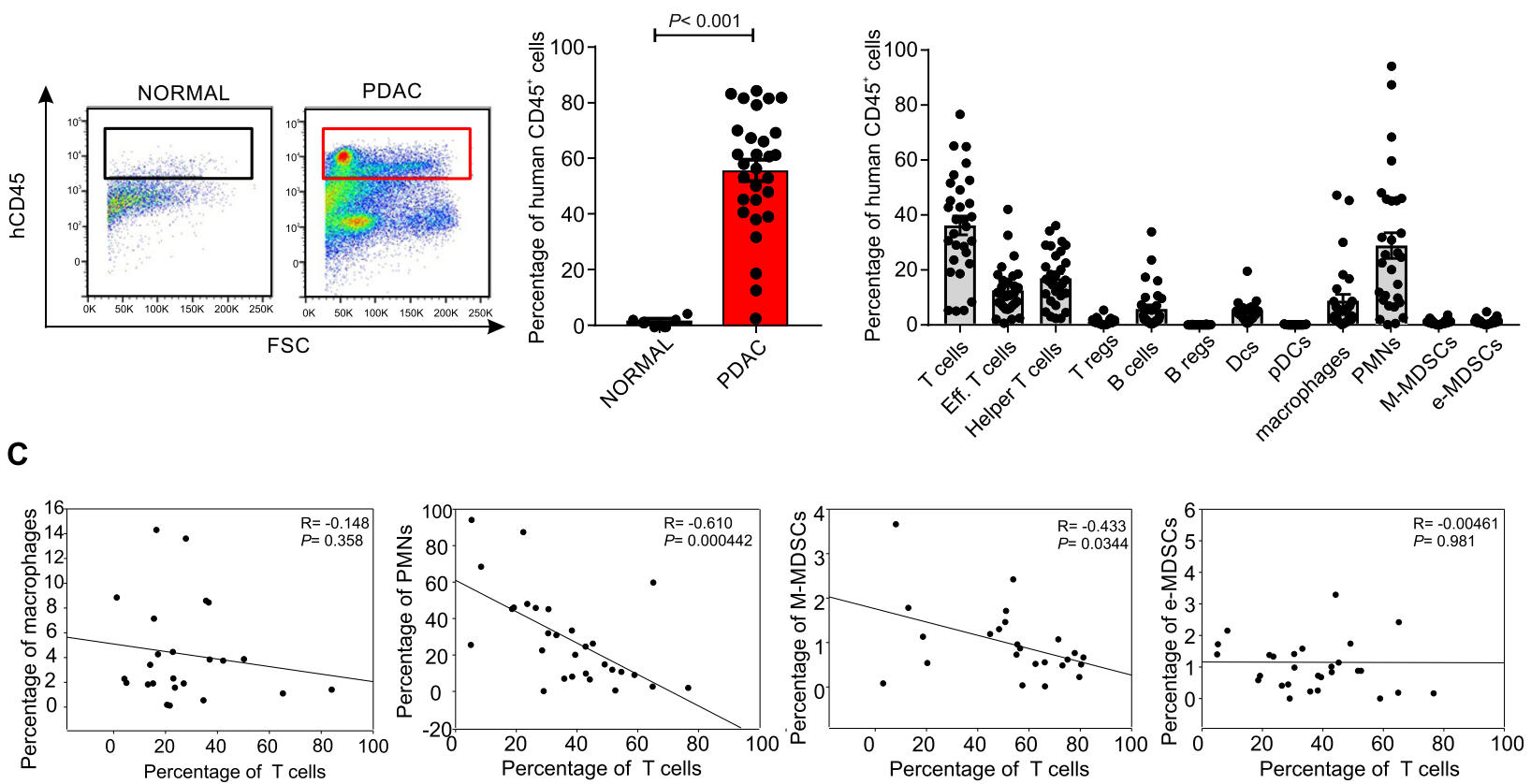

Fig. 1 Immune characterization of PDAC tumor microenvironment. a Leukocytes infiltration (CD45 cells) in normal pancreas $(n=5)$ and PDAC tissue $(n=29)$ biopsies. Statistical analysis was performed by ANOVA test. b Immune populations abundance (\% of CD45 ${ }^{+}$cells) in PDAC tissues. $\mathbf{c}$ Correlation between tumor-infiltrating T cells with either macrophages, PMNs, M-MDSCs or e-MDSCs within PDAC tissues. Correlation analysis was performed by Spearman's rank correlation

patients (stage III-IV) and 9 HDs (Fig. 2b); the last cohort consisted of 73 PDAC patients, including resectable (stage I-II, $n=21$ ) and non-resectable (stage III-IV, $n=52$ ) tumors and 28 HDs (Fig. 2c). MDSC analysis was performed using both fresh whole blood (WB) cells (Fig. 2a-b) and frozen peripheral blood mononuclear cells (PBMCs) (Fig. 2c). In fresh WB we analyzed the frequency of monocytes $\left(\mathrm{CD} 14^{+} \mathrm{CD} 15^{-} \mathrm{CD} 11 \mathrm{~b}^{+}\right)$and granulocytes (PMNs, $\left.\mathrm{CD} 15^{+} \mathrm{CD} 14^{-} \mathrm{CD} 11 \mathrm{~b}^{+}\right)$as well as the presence of MDSC1 $\left(\mathrm{CD} 14^{+} \mathrm{IL}-4 \mathrm{R}^{+}\right), \quad \mathrm{MDSC} 2 \quad\left(\mathrm{CD} 15^{+} \mathrm{IL}-4 \mathrm{R}^{+}\right), \quad \mathrm{MDSC} 3$

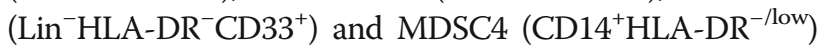
subsets (Additional file 1: Figure S3). Among frozen PBMCs, we only discriminate monocytic (MDSC1 and MDSC4) and early-stage (MDSC3) MDSCs; the assessment of PMN-MDSCs is not accurate and probably even misleading, since granulocytes (including PMN-MDSCs) are typically lost during freezing/thawing procedure. We detected a significant increase in circulating M-MDSC subsets (MDSC1 and MDSC4) in PDAC patients compared to the control group in the three independent analyses (for $\mathrm{CD} 14^{+} \mathrm{IL}-4 \mathrm{R}^{+}$cells, median value $0.19 \%$ vs. $0.57 \%, p<$ 0.001 in the first cohort, $0.18 \%$ vs. $0.59 \%, p<0.001$ in the second cohort and $2.2 \%$ vs. $4.3 \%, p=0.002$ in the third cohort; for CD14 ${ }^{+} \mathrm{HLA}_{-} \mathrm{DR}^{-/ \text {low }}$ cells, median value $0.19 \%$ vs. $0.31 \%, p=0.033$ in the first cohort, $0.08 \%$ vs. $0.32 \%, p=$ 0.042 in the second cohort and $1.78 \%$ vs. $3.25 \%, p<0.001$ in the third cohort). Moreover PMN-MDSC subset (MDSC2) was significantly increased in PDAC patients: $\mathrm{CD} 15^{+} \mathrm{IL}-4 \mathrm{R \alpha}^{+}$cells, median value $1.53 \%$ vs. $4.89 \%, p=$ 0.006 in the first cohort, $1.89 \%$ vs. $6.78 \%, p<0.001$ in the second cohort. Interestingly, PDAC patients showed an increased frequency in WB of both monocytes and granulocytes compared to HDs: for monocytes, median value was $0.94 \%$ vs. $3.15 \%, p<0.001$ in the first cohort, $0.98 \%$ vs. $3.95 \%, p<0.001$ in the second cohort; for granulocytes, median value $44.82 \%$ vs. $56.23 \%, \mathrm{p}=0.006$ in the first cohort, $47.89 \%$ vs. $62.45 \%, p<0.001$ in the second cohort. Finally, we did not observe any alteration in circulating eMDSCs (MDSC3) between HDs and cancer patients in any of the analysed cohorts.

To determine whether increased MDSC subsets could predict patient outcome, we analysed PDAC cohort 1 and 2 since these groups were homogeneously composed by poorly differentiated tumors (G3 and G4). The MDSC analysis was performed using the same sampling protocol based on fresh WB. We discovered that only MDSC2 frequency higher than optimal cutoff threshold $(9.156 \%)$ was significantly associated with a shorter patient's median overall survival (OS) (Fig. 3a) while the other analyzed MDSC subsets (MDSC1, MDSC3 and MDSC4) did not show any correlation with patients survival (Additional file 1: Figure S4). Moreover, a higher 


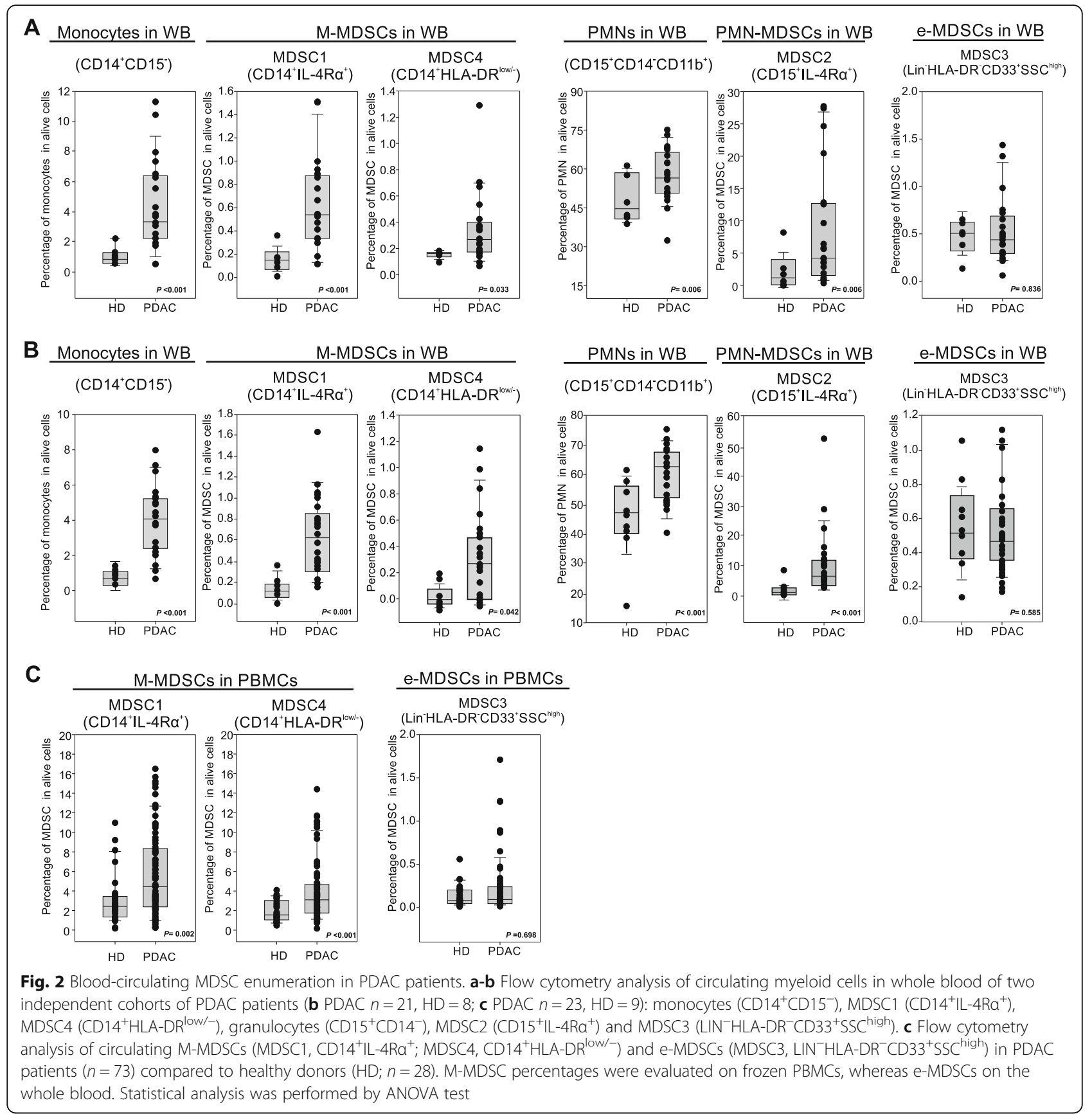

MDSC2 percentage is found in metastatic patients ( $p=$ 0.064 , Fig. 3b) and significantly discriminated patients with metastatic disease with AUC value of 0.633 ( $p=$ 0.011 , Fig. 3c) and an optimal cutoff threshold value of 9.156\% (sensitivity of $57.1 \%(95 \% \mathrm{CI}=32.6-78.6 \%)$ and a specificity of $85.7 \%(95 \% \mathrm{CI}=65.4-95 \%))$. In particular, 18 out of $24(75 \%)$ patients with MDSC2 percentage lower than the identified cutoff did not present distant cancer dissemination whereas only 6 out of 24 (25\%) patients presented clinically detectable metastases.
Conversely, 8 out of 11 (73\%) patients with MDSC2 percentage higher than the identified threshold presented metastases while 3 out of 11 (27\%) patients had no metastasis (Fig. 3d). Starting from these premises, we evaluated the power of MDSCs from frozen PBMCs in discriminating patients with metastatic disease. Only MDSC4 frequency was able to significantly discriminate non-metastatic versus metastatic tumors (Fig. 3e) with AUC value of 0.705 ( $p=0.017)$ (Fig. 3f) and a calculated threshold of $3.505 \%$ (sensitivity of $70 \%$ (95\% CI $=39.7-$ 

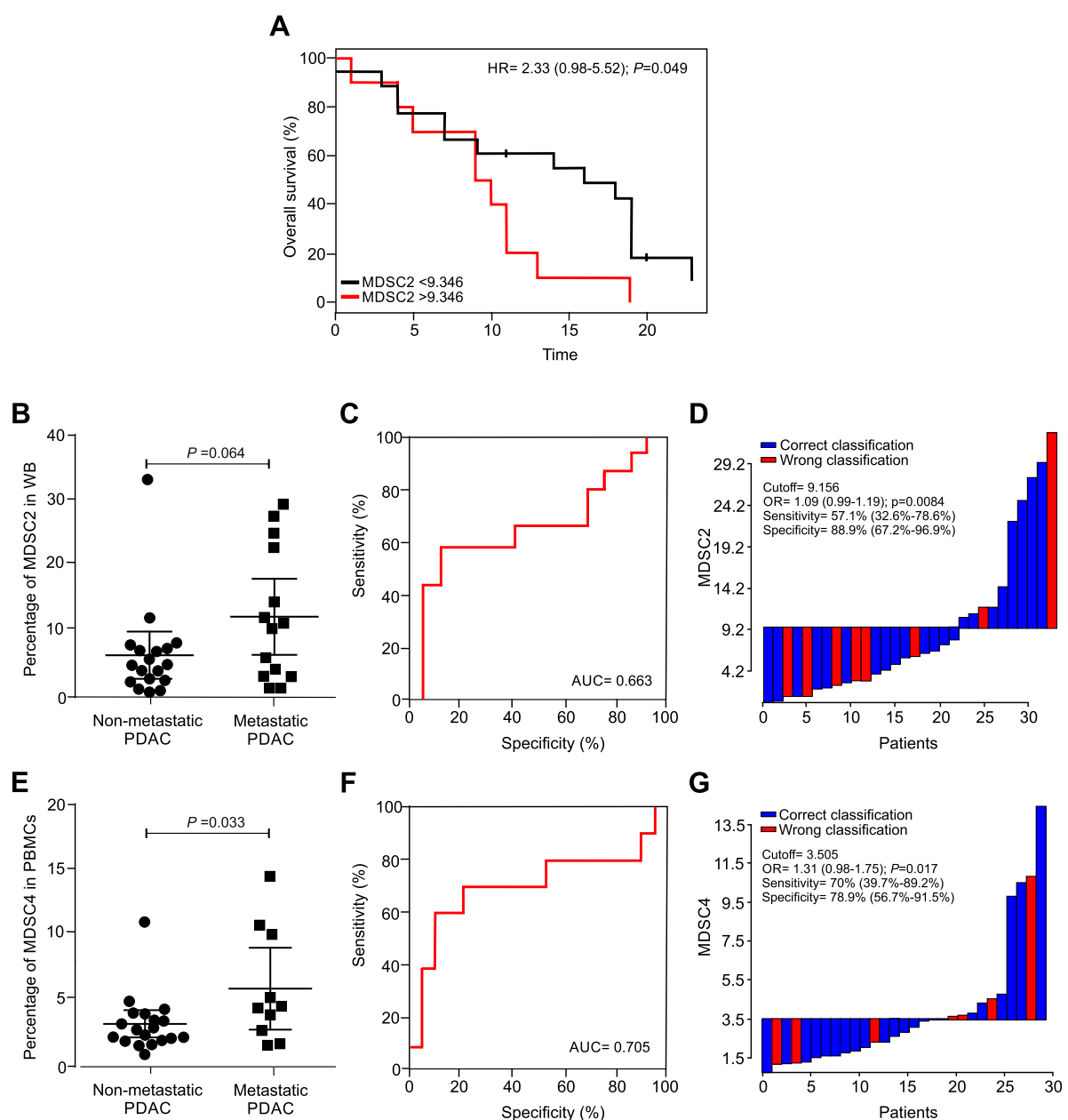

Fig. 3 Prognostic potential predictive role of MDSCs in PDAC patients. a Kaplan-Meier curves for OS by significant MDSC2 cutoff frequency in fresh whole blood samples. b MDSC2 percentages in non-metastatic and metastatic PDAC patients. Mean and 95\% confidence interval are plotted. Statistical analysis was performed by ANOVA test. c Receiver operator characteristic (ROC) curve for MDSC2 percentage in metastatic disease prediction. $\mathbf{d}$ Waterfall plot of optimal dichotomization; blue and red bars represent cases with correct or wrong classification, respectively. e MDSC4 percentage in non-recurrent and recurrent PDAC patients. Mean and 95\% confidence interval are plotted. Statistical analysis was performed by ANOVA test. $\mathbf{f}$ Receiver operator characteristic (ROC) curve for MDSC4 percentage in metastatic disease prediction. $\mathbf{g}$ Waterfall plot of optimal dichotomization, blue and red bars represent cases with correct or wrong classification, respectively

$89.2 \%)$ and a specificity of $78.9 \%(95 \% \mathrm{CI}=56.7-91.5 \%)$ ). In fact, 15 out of 18 (83.3\%) patients with MDSC4 percentage lower than the identified cutoff did not show metastases, whereas only 3 out of 18 (16.6\%) patients present a metastatic disease; on the contrary, 7 out of 11 (63.6\%) patients with MDSC4 percentage higher than the identified threshold had metastases, and 4 out of 11 (36.4\%) patients did not show metastases (Fig. 3g). Collectively, these data suggest that MDSC percentage might be a valid prognostic biomarker of advanced disease in PDAC patients, even though the selection of the biomarker is strictly dependent on sampling: when the analysis is performed on cryopreserved PBMCs, MDSC4 levels could identify patients with metastatic disease, whereas MDSC2 frequency could stratify patients with metastases when the analysis is performed on fresh blood samples.

\section{Circulating monocytes from PDAC patients induce a stronger T-cell suppression compared to PMNs resembling effective M-MDSCs}

We then evaluated in vitro the immunosuppressive properties of PMNs (isolated as $\mathrm{CD} 6 \mathrm{~b}^{+}$cells) and monocytes (isolated as $\mathrm{CD} 14^{+}$cells) freshly purified from blood samples of the second PDAC patients cohort $(n=$ 10) to confirm their MDSC-associated functional activity (cell purity was above 95\% after cell isolation, Fig. 4a). Isolated cells were co-cultured in presence of activated, cell trace-labelled allogeneic PBMCs for 4 days. As reported in Fig. 4b, at the highest T cells:myeloid cells cell 


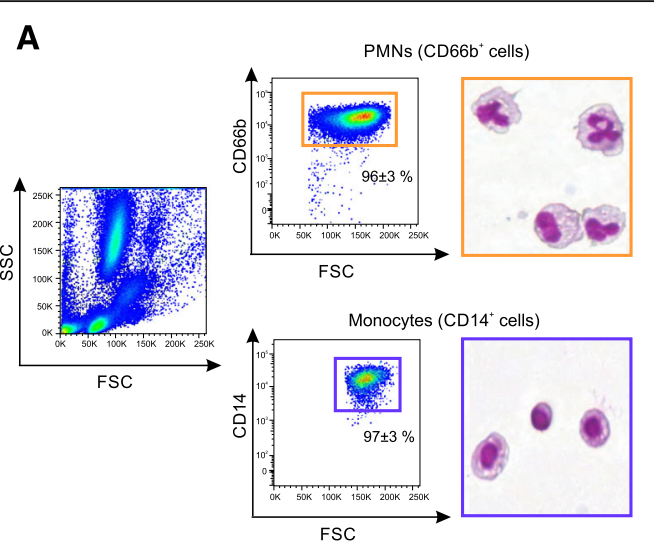

B

C

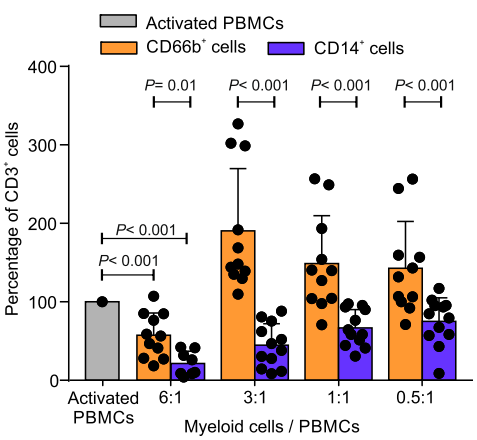

D
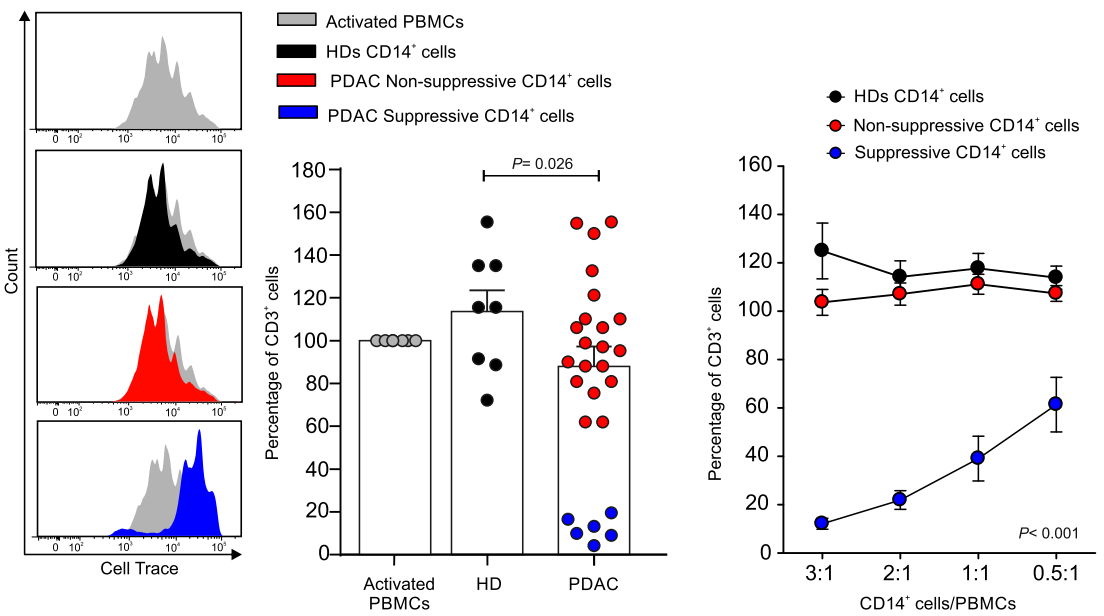

E

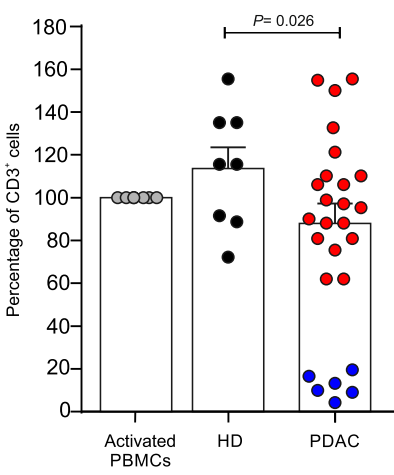

$\mathbf{F}$
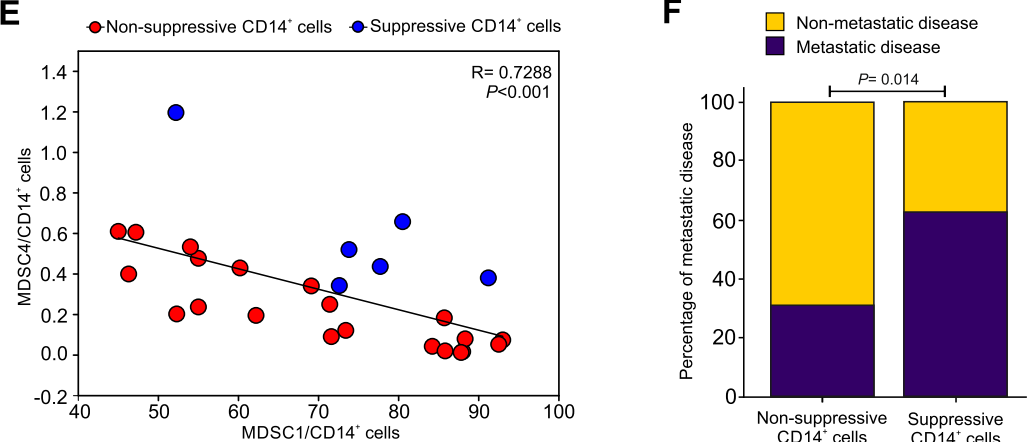

Fig. 4 Circulating monocytes from PDAC patients are able to restrain T cell proliferation in vitro. a Freshly isolated PMNs $\left(C D 66 b^{+}\right.$cells, orange box) and monocytes (CD14 ${ }^{+}$cells, blue box) from PDAC patients analysed by flow cytometry and haematoxylin-eosin staining. b Functional assay reflecting the different ability of PMNs and monocytes to affect T cells proliferation when co-cultured in vitro with CD3/CD28-activated-PBMCs at different ratios. All values are normalized on the activated PBMCs in the absence of myeloid cells (grey bar) and reported as percentage of Cell Trace $^{+} \mathrm{CD}^{+}$cells. Statistical analysis was performed by ANOVA test. c Functional assay performed (at 1:3 ratio of PBMCs:CD14 ${ }^{+}$cells) on monocytes of PDAC patients $(n=26)$ compared to HDs $(n=8)$, reported as percentage of $\mathrm{CD}^{+}$proliferating cells (right panel) and graphed as proliferation peaks of Cell Trace ${ }^{+} \mathrm{CD}^{+}$cells after the co-culture (left panel). Among all PDAC patients, "Suppressive CD14 $4^{+}$cells" (blue) and "Nonsuppressive CD14 cells" (red) were grouped based on the quantitative analysis of the in vitro immunosuppressive function. Statistical analysis was performed by ANOVA test. $\mathbf{d}$ Different ability of suppressive and non-suppressive monocytes to limit $\mathrm{CD}^{+}{ }^{+} \mathrm{T}$ cell proliferation at different cell ratios. Statistical analysis was performed by ANOVA test. e Pearson correlation between MDSC4 and MDSC1 among CD14 ${ }^{+}$cells of PDAC patients. f Pro-metastatic potential of suppressive CD14 ${ }^{+}$cells. Statistical analysis was performed by Pearson Chi-Square test 
ratio (1:6) both myeloid cell subsets showed suppressive activity, whereas only monocytes were able to restrain $\mathrm{T}$ cell proliferation at lower cell ratio (i.e. at 1:1 ratio; $p=$ 0.021 myeloid cells/PBMCs), in agreement with previous preclinical reports $[6,26]$. Therefore, these data suggest that in PDAC patients, the per cell based suppressive capacity of neutrophils is lower than the one of monocytes, as it was already observed for other tumors [27].

We further validated the immunosuppressive activity of $\mathrm{CD}_{1}{ }^{+}$cells purified from PDAC patients $(n=26)$ enrolled in the third cohort. Based on this analysis we stratified PDAC patients into two sub-groups: "suppressive PDAC" ( $n=6$, blue plots), whose monocytes were able to arrest $\mathrm{T}$ cells proliferation at different cell ratios (starting from 1:3 ratio of PBMCs:CD14 ${ }^{+}$cells), and non-suppressive/poorly suppressive PDAC $(n=20$, red plots; hereafter referred as "non-suppressive PDAC"), in which $\mathrm{CD} 14^{+}$cells did not show any inhibitory properties similarly to HD-derived monocytes $(n=8$, black plots) (Fig. 4c-d). We found that the presence of either suppressive or non-suppressive $\mathrm{CD}_{14}{ }^{+}$cells did not correlate with any MDSC subsets: M-MDSC frequency (i.e. suppressive vs. non-suppressive: MDSC1/CD14 ${ }^{+}$ cells median value 74.66 vs. $69.90, p=0.53$; MDSC4/ $\mathrm{CD}_{14}{ }^{+}$cells median value 5.78 vs. $4.09, p=0.11$ ) and the mean fluorescence intensity of IL-4R $\alpha$ expressed on MDSC1 cells (i.e. suppressive vs. non-suppressive: median value 368.83 vs. $286.19, p=0.44$ ) did not correlate with immunosuppressive activity, as well. Moreover, we did not identify any clinical parameter able to discriminate the suppressive and not suppressive group of patients (i.e. those with immunosuppressive or not suppressive CD14 ${ }^{+}$cells). In fact, cell counts were not significantly different between the analyzed groups (suppressive vs. non-suppressive): WBE $\left[10^{9} / \mathrm{L}\right]$ median value 5.89 vs. $6.08, p=0.76$; neutrophils $\left[10^{9} / \mathrm{L}\right]$ median value 3.845 vs. 3.749, $p=0.86$; monocytes $\left[10^{9} / \mathrm{L}\right]$ median value 0.265 vs. $0.344, p=0.16$; lymphocytes $\left[10^{9} / \mathrm{L}\right]$ median value 1.58 vs. $1.65, p=0.58$. Interestingly, we observed an inverse correlation comparing the frequency of MDSC1 and MDSC4 among monocytes (Fig. 4e), which allowed to distinguish the $80 \%$ of suppressive PDAC samples towards non-suppressive samples: suppressor monocytes showed simultaneously discrete amount of both MDSC-1 (MDSC1/CD14 $\left.{ }^{+}>70 \%\right)$ and MDSC-4 (MDSC4/ $\mathrm{CD}_{1} 4^{+}>0.2 \%$ ), suggesting that both cell populations play a critical role in promoting a functional inhibition of $\mathrm{T}$ cell proliferation. Furthermore, the presence of suppressive $\mathrm{CD}_{14}{ }^{+}$cells were able to significantly cluster metastatic versus non-metastatic patients: in fact, 11 out of 17 patients that exhibited suppressive monocytes (64.7\%) presented a metastatic disease while this was found only in 8 out of 29 patients with non-suppressive monocytes (27.6\%). Conversely, only 6 out of 17 patients presented a suppressive profile (35.3\%) whereas 21 out of 29 patients with a non-suppressive profile (72.4\%) exhibited a nonmetastatic disease (Fig. 4f). Collectively, these data highlight that suppressive monocytes have a pro-metastatic potential.

\section{Immunosuppressive PDAC-derived CD14 ${ }^{+}$cells mainly activate a STAT3/arginase 1 axis}

In order to define the molecular network relevant for $\mathrm{CD}_{14}{ }^{+}$cell immunosuppression, we performed a genomewide mRNA expression profiling on purified monocytes isolated from 3 suppressive and 4 non-suppressive PDAC patients. First of all, we compared the gene profiles of PDAC-derived monocytes against three independent public datasets of normal circulating $\mathrm{CD} 14^{+}$cells isolated from HDs (GSE60601, GSE64480 and GSE13899) demonstrating a specific cancer-related signature, as the hierarchical gene clustering revealed different patterns of expression between the two groups (Additional file 1: Figure S5A). Indeed, by using the gene set enrichment analysis (GSEA) of hallmarks of cancer, the differentially expressed genes were enriched in categories involved in: TNF $\alpha$ signaling via NF- $\mathrm{kB}$, inflammatory response, IL6 JAK/STAT3 signaling and apoptosis categories (Additional file 1: Figure S5B). These results are in agreement with our recent findings [12] and indicate how cancer cells alter the normal monopoiesis favouring the development of $\mathrm{CD}_{1} 4^{+}$cells with cancer-related imprint. To elucidate further this cancer-driven reprogramming, we compared the gene profile of suppressive towards non suppressive PDAC monocytes by clustering genes according to their expression levels, demonstrating that immunosuppressive monocytes had a distinctive gene signature (Fig. 5a). The comparative analysis identified differences in the expression of genes involved in metabolism, cell cycle, signaling and structural components (Fig. 5b). Considering the structural constituents category, suppressive $\mathrm{CD} 14^{+}$cells showed a greater expression in FBN2, TSPAN16, LEPR, CLTA and $C D 163$ that are normally associated with classic monocytes. In particular, CD163 expression is strongly regulated by IL- 6 and IL-10 that are two of the main inflammatory mediators in PDAC patients' sera [12, 21]. Moreover, the CD163 cleaved form (sCD163), released by monocytes/macrophages, was reported to inhibit $\mathrm{T}$ cell proliferation, underlying its potential involvement in immune evasion [28]. Suppressive monocytes showed also an altered cell cycle-associated gene signature, as well as a complex signaling-related gene enrichment. Among cell cycle cluster, we found the expression of $C A S P 2$, recently described as a regulating key of myeloid progenitor differentiation [29]; AKAP9, involved in c-AMP-dependent suppression on LPS-activated macrophages and NLRP1, described to impair $\mathrm{T}$ cell responses [30]. In the signaling 
A

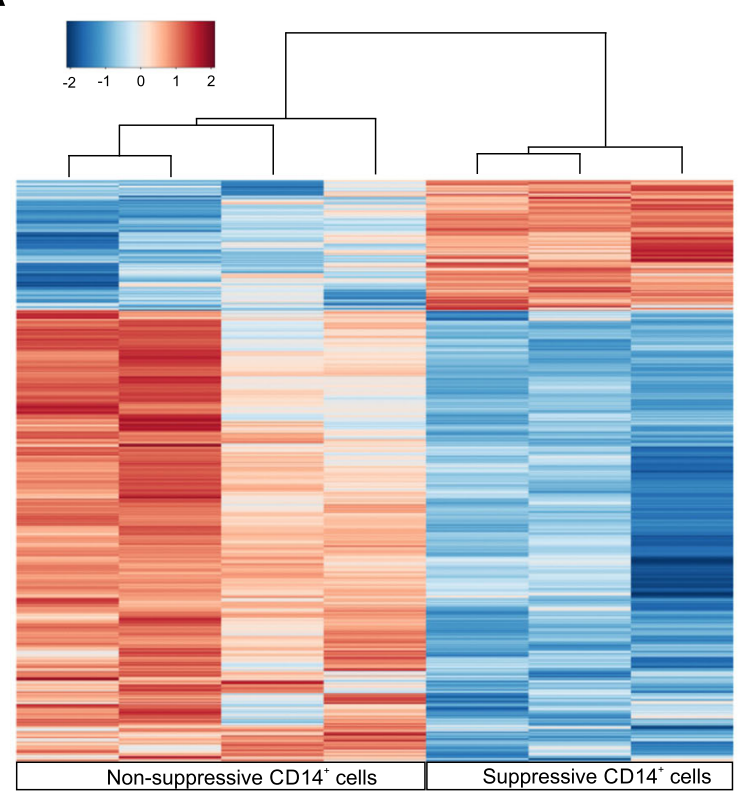

B
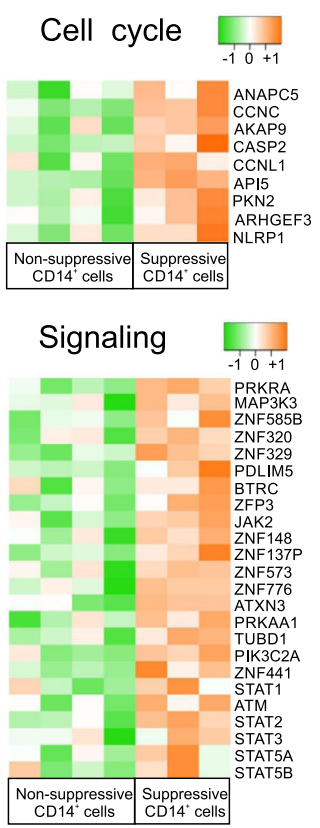
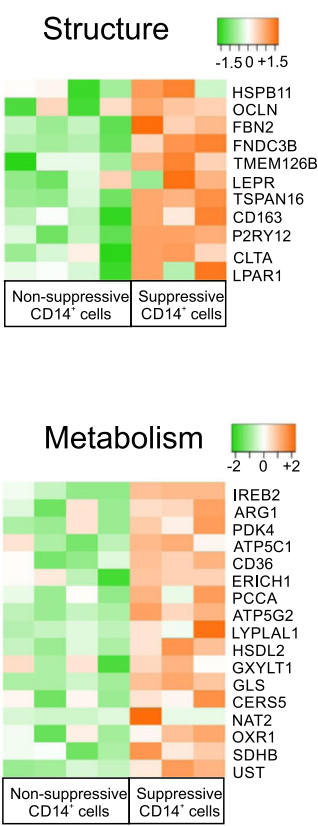

C

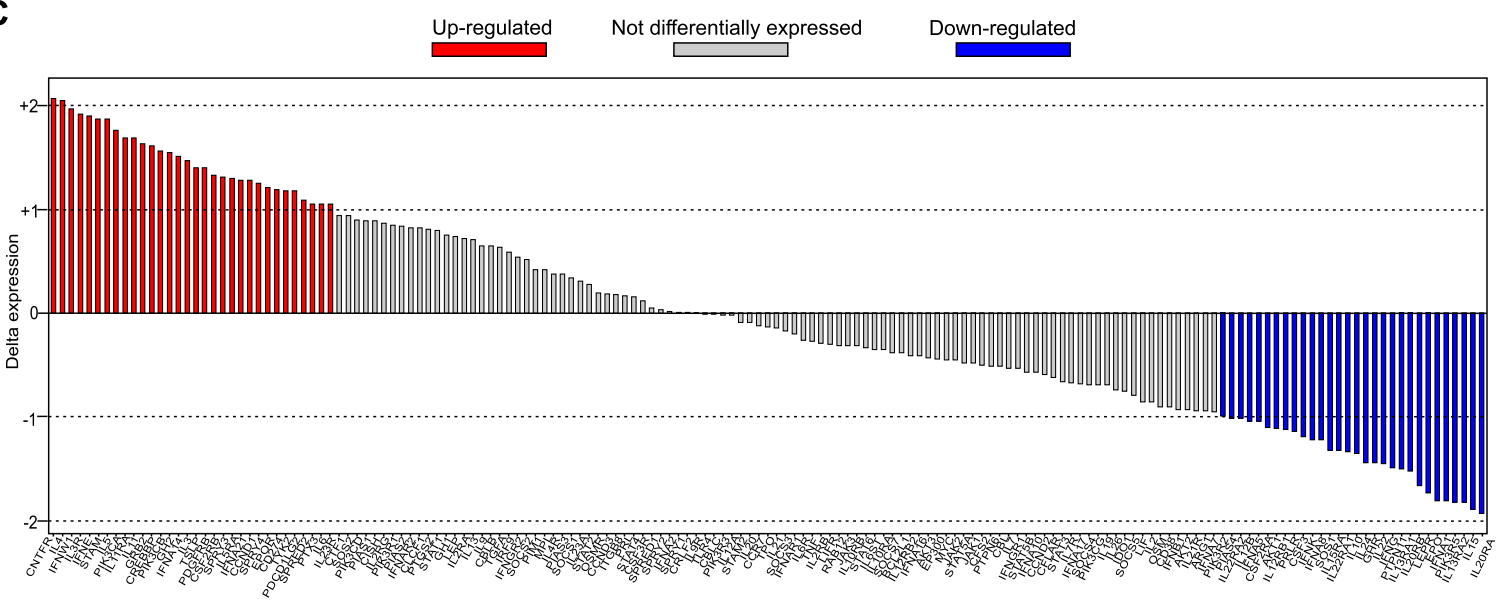

D

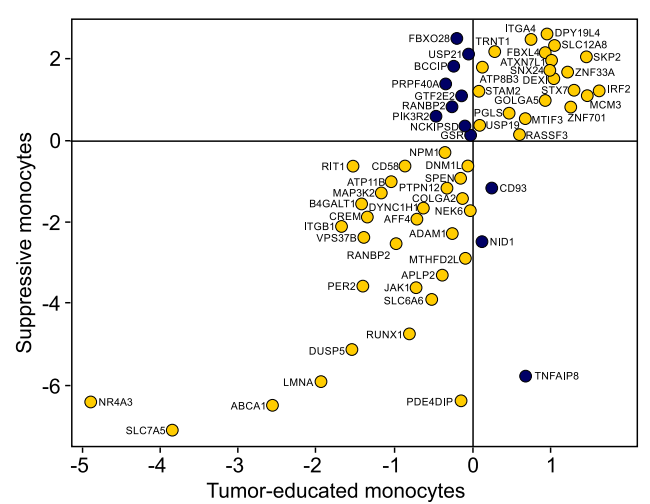

E

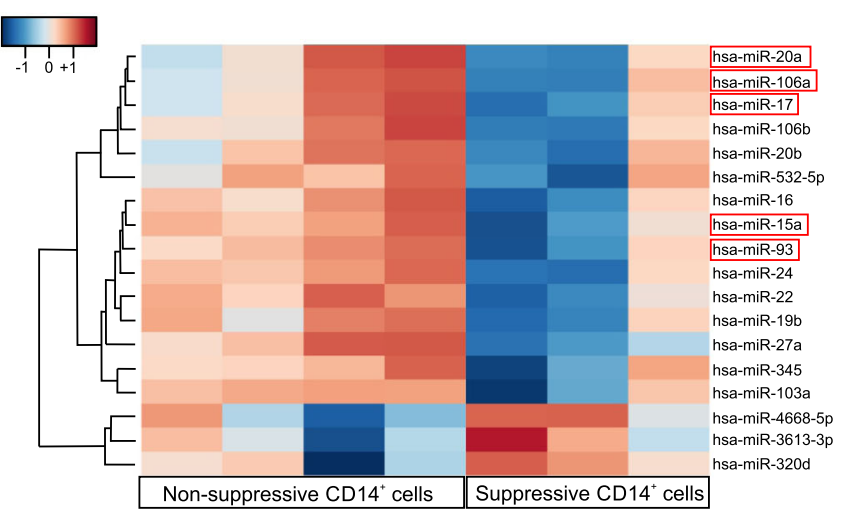

Fig. 5 (See legend on next page.) 
(See figure on previous page.)

Fig. 5 Gene profiling of suppressive CD14 $4^{+}$cells isolated from PDAC patient. a Supervised clustering of suppressive and not suppressive monocytes arrays using 1119 differentially expressed genes ( $F D R<0.05$ and absolute fold change $>2$ ). $\mathbf{b}$ Clustering of cell cycle, structure, signaling and metabolism in suppressive- and not suppressive monocytes (absolute fold change $>2$; FDR $<20 \%$ ). c Difference in expression between suppressive monocytes isolated from PDAC patients and human BM-MDSCs samples for genes in JAK/STAT Signaling Pathway. d Dot plot of log fold change demonstrating common (yellow plots) or different (purple plots) gene expression modulation between differentially expressed signature of either tumor-educated or suppressive monocytes to related controls. e miRNAs-expression profile of suppressive and non-suppressive CD14 $4^{+}$cells isolated from PDAC patients using 19 differentially expressed miRNAs (FDR $<0.05$ and absolute fold change $>2$ )

category, we identified the expression of several zincfinger protein-coding genes (ZFP3, ZNF585B, ZNF320, ZNF329, ZNF148, ZNF137P, ZNF573, ZNF776 and $Z N F 441)$, as well as the different pattern of expression of $M A P 3 K 3, P R K R A, J A K 2$ and different components of the STAT family (STAT1, STAT2, STAT3, STAT5A, STAT5B and STAT6) that have been already defined as MDSCassociated transcriptional factors [9]. In the metabolism group, we identified several genes potentially linked to immunosuppression [31]: fatty acid and lipoprotein metabolism-related genes, such as CD36, LYPLA1 and CERS5; energy (ATP) metabolism-associated genes, such as $A T P 51 C$, ATP5G2 and SDHB; glucose metabolismassociated genes, such as PDK4 and GXYLT1, as well as hormone and water soluble vitamins metabolismassociated genes (i.e. HSDL2 and PCCA respectively). Finally, we identified different genes involved in both amino acid metabolism, such as ERICH1, GLS, CTSC and ARG1 and amino acid modifying enzymes, such as NAT2, UST and OXR1. To understand the depth of cancerinduced monocyte reprogramming towards MDSCs, we compared suppressive $\mathrm{CD} 14^{+}$cell gene profiles to gene signatures of human bone marrow (BM) derived MDSCs (BM-MDSCs, $n=8$ independent donors) obtained by in vitro differentiation of $\mathrm{BM}$ cells in presence of a cytokine cocktail composed by G-CSF and GM-CSF, as previously reported [13] (Additional file 1: Figure S5C). Despite the phenotypic differences and the expected variances in their isolation and generation, BM-MDSCs and cancer patient immunosuppressive monocytes displayed a shared signature (not-differentially expressed genes, Fig. 5c) characterized by genes such as PTGS2, SOCS2, TNF, IDO1, CD38 and ARG1, all related to immune regulation. Interestingly, they also shared expression levels of AKT3, JAK1, JAK3, STAT1, STAT4, STAT5, STAT6 and STAT3, suggesting a common signaling network among these myeloid cells, as well as the same expression of CFLAR, which we recently reported as an important candidate for driving the acquisition of the immunosuppressive program in monocytes [12].

Notably, we identified a cluster of genes that are equally modulated in both suppressive monocytes and tumoreducated monocytes (recently described in [32]), suggesting a common tumor-dependent re-programming circuit (Fig. 5d). Among the most significant genes we identified
SKP2, IRF2 and MCM3, all related to tumor progression and metastases [33-35]. In agreement with these shared cues, 5 signaling pathways (MAPK, JAK-STAT, p53, VEGF and PI3K) that were not significantly different between immunosuppressive monocytes and tumoreducated monocytes, were observed; however, we found other signaling pathways uniquely upregulated in suppressive monocytes NF-kB, TGF $\beta$, TNF $\alpha$, Hypoxia, TRAIL and EGFR (Additional file 1: Figure S5D). Collectively, these data pinpoint suppressive monocytes as a peculiar subgroup of tumor-educated monocytes.

Finally, we integrated the transcriptome with a complete miRNAs profiling analysis of suppressive vs. non-suppressive PDAC CD14 ${ }^{+}$cells, using the same samples. The hierarchical clustering highlighted only 18 miRNAs that were differentially expressed between the two experimental groups (Fig. 5e). Surprisingly, among the down-regulated miRNAs in the suppressive CD14 ${ }^{+}$ cells $(n=15)$, we identified mir-17, mir-20a, mir-93, mir106 and mir-15a that were reported to directly inhibit STAT3 [36, 37]. Indeed, these miRNAs are part of the 50 validated miRNAs able to bind the 3 '-UTR region of STAT3 [37]. Therefore, these data allowed us to hypothesize that gain of suppressive function in MDSC could be partly dependent on the activation of a STAT3dependent gene transcription.

To prove the role of STAT3 among transcriptional factors driving MDSC function in PDAC, we first demonstrated an enhanced expression of the $\mathrm{Tyr}_{705^{-}}$ phosphorylated STAT3 (p-STAT3) in suppressive monocytes (Fig. 6a). Notably, treatment with Stattic, a specific small-molecule inhibitor of STAT3, significantly abrogated the suppressive activity of $\mathrm{CD} 14^{+}$cells, while it had no effects in non-suppressive monocytes, confirming the role of STAT3-driven program in MDSCassociated function (Fig. 6b). These results are consistent with data from Vasquez-Duddel et al. that demonstrated the therapeutic impact of Stattic on controlling MDSC function in head and neck squamous cell carcinoma [14]. Since p-STAT3 is able to bind different sites on the ARG1 promoter to favor its transcription, we focused our next analyses on ARG1 expression. We measured ARG1 protein levels in both suppressive and nonsuppressive CD $14^{+}$cells by flow cytometry and immunofluorescence (IF). We demonstrated that CD $14^{+} \mathrm{ARG} 1^{+}$ 


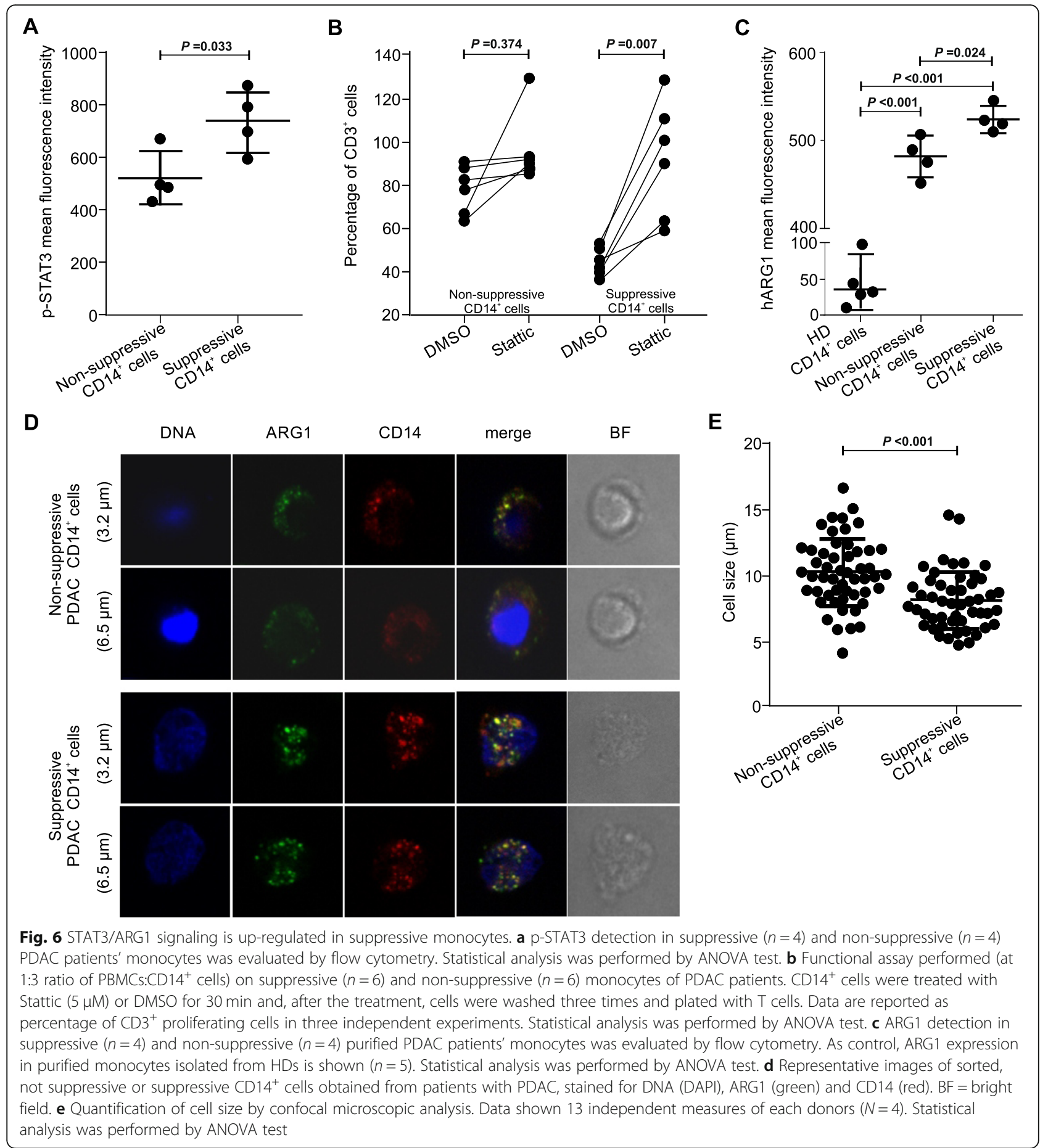

cells were significantly increased in cancer patients as compared to the HDs (Additional file 1: Figure S6A). However, they were not significantly different among suppressive vs. non-suppressive groups (median value $50.9 \pm$ 3.25 vs. $48.6 \pm 4.38 ; p=0.76$ ). We then measured the distribution and intensity of ARG1 by IF and we found a higher amount of ARG1 in suppressive than in nonsuppressive monocytes (Fig. 6c). Additionally, confocal analysis showed a different pattern of ARG1 distribution, with the suppressive $\mathrm{CD} 14^{+}$cells being smaller and with diffused and less clustered ARG1-containing granules (Fig. 6d). By Z-stack analysis, suppressive CD14 ${ }^{+}$cells shared a significant smaller size (Fig. 6e), suggesting that immunosuppressive monocytes, that resemble M-MDSCs, may be clearly distinguished from monocytes present under steady-state hematopoiesis as small, $\mathrm{ARG} 1^{+} \mathrm{CD} 14^{+}$cells. 


\section{Discussion}

Since the first identification of MDSCs at the end of 1970, both ontogeny and classification of those cells have been controversial [8]. In fact, while MDSCs share morphological features with conventional monocytes and granulocytes, they own the ability to dampen immune responses. Different reports have tried to identify unique MDSCs markers, but the newly identified subsets were rarely tested for inhibitory function, consequently missing the main characteristic to define those cells as MDSCs. Thus, following the recently published recommendations for MDSC characterization [15], we applied flow-cytometry technique to discriminate circulating MDSCs in PDAC patients and tested their ability to restrain $\mathrm{T}$ cell proliferation, in vitro. Interestingly, we unveiled an overall increased frequency of monocytes and PMNs in PDAC patients, probably as a consequence of tumor-induced inflammation. Among these cells, both $\mathrm{M}$ - and PMN-MDSC subsets were expanded in PDAC patients, both in the blood and tumor tissue, and overall, they were able to control $\mathrm{T}$ cell proliferation. Moreover, we demonstrated that $\mathrm{CD} 14^{+}$cells isolated from PDAC patients have a distinctive gene signature as compared to the monocytes isolated from HD suggesting that, during cancer progression, monocytes activate specific transcriptional programs that can be, in turn, used as potential biomarkers. Among patient-derived myeloid cells, we found $\mathrm{CD} 14^{+}$cells as the most potent myeloid subset to halt $\mathrm{T}$ cell proliferation, in line with previous preclinical data [26]. Notably, the functional assay allowed us to identify two distinct M-MDSC subgroups, i.e. suppressive and non-suppressive CD14 $4^{+}$cells. This cell discrimination was not connected with changes on the type and intensity of MDSC-associated markers but relied exclusively on functional suppressive ability mainly mediated by STAT3 activation. Therefore, functional tests cannot be considered as an adjunct to MDSC identification but, on the contrary, have a prioritizing role for definition of MDSC subsets. However, since standardizing functional tests among different laboratories might represent a challenging hurdle [38], we focused our subsequent efforts in identifying genes and molecular pathways that can represent clues to their immunosuppressive ability.

Further genetic analysis on M-MDSCs highlighted several other up-regulated targets linked to different molecular pathways, including the amino acid metabolism. In this gene-cluster we identified $A R G 1$, which has been already associated to MDSC function in both tumorbearing mice and cancer patients [39-41]. We recently reported that ARG1 has a hierarchical role in generating an immunosuppressive tumor microenvironment among the L-arginine metabolizing enzymes; indeed, myeloid cells expressing high levels of the inducible nitric oxide synthase (iNOS) enzyme (i.e. TNF $\alpha$ - and NO-producing dendritic cells) actively sustained anti-tumor $\mathrm{T}$ cells response and were counteracted by ARG1 activation in tumor-associated macrophages [42]. Differently, Larginine deprivation, due to ARG1-expressing cells, strongly compromised both $\mathrm{T}$ cell proliferation and activation [43]. Moreover, ARG1-derived products, i.e. polyamines, could directly contribute to the generation of tolerogenic DCs through IDO1 phosphorylation, thus sustaining the immunosuppressive tumor microenvironment [44]. For the first time, our data demonstrated that ARG1 is expressed in human cancer-programmed monocytes, with the suppressive $\mathrm{CD} 14^{+}$monocytes expressing higher amount of the protein and presenting a unique pattern of staining, making them trackable from other circulating monocytes. Notably, circulating monocytes may acquire both ARG1 expression and MDSCassociated functions after tumor-derived exosomes uptake suggesting exosomes as tumor-derived cues to reprogram monocytes into immunosuppressive cells $[45,46]$. Thus, ARG1-targeting approaches, using either ARG1 inhibitors or small molecules interfering with ARG1 transcription, such as AT38 or Stattic [14, 47-49], may offer a potential therapeutic options to the most aggressive form of PDAC. We think that targeting ARG1, with the new upcoming generation of engineered MDSC-specific nanoparticles, will be of great interest for many cancer types to unleash anti-tumor immunity. To this aim, we recently demonstrated the ability of newly designed lipid nanocapsules to vehicle, in vivo, chemotherapeutic agents exclusively to M-MDSCs, enhancing the therapeutic impact of immunotherapy [50].

Only in the recent past, PDAC was deeply interrogated at the genetic level, therefore revealing the presence of different molecular subtypes and, among them, an immune subgroup [51]. Moreover, the presence of a marked $\mathrm{T}$ cell-response against neo-antigen in PDAC microenvironment was associated to a better patients' outcome and long term survival [22]. Finally, the recent efficacy of a combinatorial therapy, based on chemotherapy and immune checkpoint inhibition, in a mouse model of PDAC, open up to the development of several immunotherapeutic approaches that we hope could be successfully translated into the clinic, in the next years [52-54]. Of relevance are also recent results on TGF $\beta$-targeted therapy showing synergism with anti-PDL1-based treatment by dampening the tumor immunosuppressive microenvironment generated by peri-tumoral fibroblasts and favoring $\mathrm{T}$ cell trafficking to the tumor [55]. In many cancer patients, the lack of efficacy of several immunotherapeutic approaches, is related to the presence of an immunosuppressive network, mainly composed by MDSCs and macrophages that interferes with $\mathrm{T}$ cell trafficking and activation [24]. In line with these observations, our analysis of PDAC tumor- 
infiltrating leukocytes confirmed the negative association of these cells with $\mathrm{T}$ lymphocyte accumulation and activation. Therefore, we think that an effective immunotherapeutic approach, in PDAC patients, should combine the induction or transfer of tumorspecific $\mathrm{T}$ cells with the elimination of immunosuppressive cells. To this aim, several strategies might be explored to generate effective therapies, such as the use of low-dose chemotherapy, able to abrogate MDSC proliferation, accumulation and function [56, 57]; or, specific antibodies that limit myeloid migration into the tumor, such as monoclonal antibodies to CSF-1 receptor (CSF-1R), or antibodies to CCR2 and to CXCR4 [58], as well as the use of antibodies able to restrain tumor-induced inflammation (i.e. anti-IL-6 antibody).

\section{Conclusion}

In conclusion, patient-derived M-MDSCs, identified as suppressive $\mathrm{CD}_{14}{ }^{+}$cells, showing distinctive cytological features (a smaller size), functional properties (the ability to abrogate $\mathrm{T}$ cells) and gene signatures (i.e. activation of STAT3/ARG1 pathway) represent a peculiar branch within the complexity and heterogeneity of monocyte population found in tumors. We do not believe that MDSCs definition is an outdated concept, as recently postulated [59], but it rather defines a myeloid cell subset with unique properties, as we demonstrated in this work. It remains to be determined the tumorderived factors that contribute to the development of this suppressive monocytes and we believe that singlecell technologies and fate mapping will help to reveal more information. In this regards, our data open a new insight in PDAC and in MDSC biology that may lead to more specific diagnosis and treatment for this lethal disease.

\section{Supplementary information}

Supplementary information accompanies this paper at https://doi.org/10. 1186/s40425-019-0734-6.

Additional file 1. Supplementary methods. Figure S1. Gating strategy to identify tumor-infiltrating leukocytes. Figure S2. Immune characterization of PDAC tumor microenvironment. Figure S3. Gating strategy to identify circulating MDSCs in fresh whole blood. Figure S4. Prognostic potential role of MDSCs in PDAC patients. Figure S5. Gene signature of $\mathrm{CD}_{1} 4^{+}$cells isolated from PDAC patients. Figure S6. Enumeration of circulating $\mathrm{CD} 14^{+} \mathrm{ARG} 1^{+}$cells in PDAC patients.

\footnotetext{
Abbreviations

Ab: Antibody; APC: Allophycocyanin; APC-Cy7: Allophycocyanin cyanine 7; ARG1: Arginase-1; ATP: Adenosine triphosphate; AUC: Area under the roc curve; BM: Bone marrow; Bregs: Regulatory B cells; C/EBP: CCAAT-enhancerbinding proteins; C-AMP: Cyclic adenosine monophosphate; CCR: C-C chemokine receptor; CD: Cluster of differentiation; CFLAR: Caspase-8 and FADD like apoptosis regulator; $\mathrm{CO}_{2}$ : Carbon dioxide; CSF: Colony stimulating factor; CTLA-4: Cytotoxic T-lymphocyte antigen 4; CXCR: C-X-C chemokine receptor; DC: Dendritic cells; EDTA: Ethylenediaminetetraacetic acid; FADD: Fas-associated protein with death domain; FBS: Fetal bovine serum;
}

FCR: FC receptor; FITC: Fluorescein isothiocyanate; FoxP3: Forkhead box P3; GCSF: Granulocyte colony-stimulating factor; GM-CSF: Granulocytemacrophage colony-stimulating factor; HD: Healthy donors; HEPES: 4-(2hydroxyethyl)-1-piperazineethanesulfonic acid; IDO1: Indoleamine 2,3dioxygenase 1; IF: Immunofluorescence; IL: Inteleukin; iNOS: Inducible nitric oxide synthase; JAK: Janus kinase; LPS: Lipopolysaccharide; M: Monocytic cells; MDSC: Myeloid-derived suppressor cells; MGG: May-Gruwald-Giemsa; mir: microRNA; NF-kB: Nuclear factor kappa-light-chain-enhancer of activated B cells; OS: Overall survival; PBMCs: Peripheral blood mononuclear cells; PDAC: Pancreatic ductal adenocarcinoma; pDC: Plasmacitoid DCs; PE: Phycoerythrin; PE-Cy7: Phycoerythrin cyanine 7; PMN: Polymorphonuclear cells; p-STAT3: Tyr ${ }_{705}$-phosphorylated STAT3; RNA: Ribonucleic acid; RNS: Reactive nitrogen species; ROC: Receiver operating characteristic; ROS: Reactive oxygen species; STAT: Signal transducer and activator of transcription; TGF- $\beta$ : TGFtransforming growth factor beta; TNFa: Tumornecrosis factor alfa; Treg: Regulatory T cells; UTR: Untranslated region

\section{Acknowledgments}

The authors thank the patients who contributed samples to the study and research nurses for collecting of patients' samples. We thank Sara Sandri, Giovanna Ferrarini, Irene Pagliarello, Giulio Fracasso and Tiziana Cestari (Immunology Section of University of Verona); Boris Rusev, Nicola Sperandio, Giada Bonizzato and Sonia Grimaldi (ARC-Net Centre for Applied Research on Cancer) for technical assistance. We thank the Italian Foundation for Pancreatic Diseases Research ONLUS (Fondazione Italiana per la Ricerca sulle Malattie del Pancreas).

\section{Authors' contributions}

Conceptualization: RT; AF, SSartori, SC, SU and VB. Methodology: RG, SSolito, SM and SU. Formal analysis: RT, AF, SSartori, LC, SC, FDS, FH, OP, CA, SSolito, LP, CC and GP. Investigation: RT, AF, SSartori, LC, RG, SSartoris, SP, VC and SU. Resources: SP, RS, RL, CB and AS. Writing: RT; AF, SSartori, SSartoris, SU and VB. Supervision: SU and VB. Funding Acquisition: CB, SU and VB. All authors read and approved the final manuscript.

\section{Funding}

This work was supported by VB grants (18603 and 12182 that refers to Special Program Molecular Clinical Oncology 5 per mille) and fellowships (Francesco De Sanctis, AIRC/FIRC 2013; Alessandra Fiore, AIRC/FIRC 2017 and Rosalinda Trovato, AIRC/FIRC 2018) from Italian Association for Cancer Research (AIRC) and by grants provided by: the Cancer Research Institute (Clinic and Laboratory Integration Program, CLIP_2017), Euronanomed III (Joint Traslational Call_2017, Project Resolve), Cariverona Fondation (Project call, 2017) and Qatar National Priority Research Program 2017, Project: NPRP11S-1211-170086). This work was supported by CB grant (RF-201602361940) from University of Verona and SU grant (21509) from Italian Association for Cancer Research (AIRC).

\section{Availability of data and materials}

The datasets used and/or analyzed during the current study as well as reagents and samples are available from the senior authors on reasonable request and under material transfer agreement.

\section{Ethics approval and consent to participate}

Informed consent was obtained from all participants and the study was approved by the Ethics Committee: for the use of PDAC and HD PBMCs, Prot. 25978, Prog. 2172 on 29/05/2012 from Comitato Etico per la sperimentazione clinica delle province di Verona e Rovigo, principal investigator: Aldo Scarpa; for the use of $\mathrm{Lin}^{-}$cells from fresh BM aspirate samples Prot. 2014/101 on 15/12/2014 from Comitato Etico per la sperimentazione clinica della provincia di Padova principal investigator: Susanna Mandruzzato; for the use of PBMCs isolated from buffy coats Prot. 24114 on 16/05/2017 from Comitato Etico per la sperimentazione clinica delle province di Verona e Rovigo, principal investigator: Vincenzo Bronte.

Consent for publication

Not applicable. 


\section{Author details}

'University Hospital and Department of Medicine, Section of Immunology, University of Verona, Verona, Italy. ${ }^{2}$ Present Address: Max Planck Institute of Biochemistry, Martinsried, Germany. ${ }^{3}$ Department of Computer Science, University of Verona, Verona, Italy. ${ }^{4}$ Institute of Oncology Research, Bellinzona, Switzerland. ${ }^{5}$ General and Pancreatic Surgery, Pancreas Institute, University of Verona, Verona, Italy. ${ }^{6}$ Medical Oncology, Fondazione Policlinico Universitario Agostino Gemelli, IRCCS, Rome, Italy. ${ }^{7}$ Faculty of Medicine and Surgery, Università Cattolica del Sacro Cuore, Rome, Italy. ${ }^{8}$ Department of Department of Diagnostic and Public Health, University of Verona, Verona, Italy. ${ }^{9}$ ARC-Net Centre for Applied Research on Cancer, University and Hospital Trust of Verona, Verona, Italy. ${ }^{10}$ Department of Surgery, Oncology and Gastroenterology, Section of Oncology and Immunology, University of Padova, Padova, Italy. ${ }^{11}$ Present Address: Centro Piattaforme Tecnologiche (CPT), University of Verona, Verona, Italy. ${ }^{12}$ Istituto Oncologico Veneto IOV-IRCCS, Padova, Italy.

Received: 12 June 2019 Accepted: 5 September 2019

Published online: 18 September 2019

\section{References}

1. Groot VP, et al. Patterns, timing, and predictors of recurrence following pancreatectomy for pancreatic ductal adenocarcinoma. Ann Surg. 2018; 267(5):936-45.

2. Neoptolemos JP, et al. Comparison of adjuvant gemcitabine and capecitabine with gemcitabine monotherapy in patients with resected pancreatic cancer (ESPAC-4): a multicentre, open-label, randomised, phase 3 trial. Lancet. 2017;389(10073):1011-24.

3. Cid-Arregui A, Juarez V. Perspectives in the treatment of pancreatic adenocarcinoma. World J Gastroenterol. 2015;21(31):9297-316.

4. Incio J, et al. Obesity-induced inflammation and Desmoplasia promote pancreatic Cancer progression and resistance to chemotherapy. Cancer Discov. 2016;6(8):852-69.

5. Rielland M, et al. Senescence-associated SIN3B promotes inflammation and pancreatic cancer progression. J Clin Invest. 2014;124(5):2125-35.

6. Dolcetti L, et al. Hierarchy of immunosuppressive strength among myeloidderived suppressor cell subsets is determined by GM-CSF. Eur J Immunol. 2010;40(1):22-35.

7. Bayne LJ, et al. Tumor-derived granulocyte-macrophage colony-stimulating factor regulates myeloid inflammation and T cell immunity in pancreatic cancer. Cancer Cell. 2012;21(6):822-35.

8. Talmadge JE, Gabrilovich DI. History of myeloid-derived suppressor cells. Nat Rev Cancer. 2013;13(10):739-52.

9. De Sanctis F, et al. MDSCs in cancer: conceiving new prognostic and therapeutic targets. Biochim Biophys Acta. 2016;1865(1):35-48.

10. Gabrilovich DI, Ostrand-Rosenberg S, Bronte V. Coordinated regulation of myeloid cells by tumours. Nat Rev Immunol. 2012;12(4):253-68.

11. Ugel S, et al. Tumor-induced myeloid deviation: when myeloid-derived suppressor cells meet tumor-associated macrophages. J Clin Invest. 2015; 125(9):3365-76.

12. Fiore $A$, et al. Induction of immunosuppressive functions and NF-kappaB by FLIP in monocytes. Nat Commun. 2018;9(1):5193.

13. Marigo I, et al. Tumor-induced tolerance and immune suppression depend on the C/EBPbeta transcription factor. Immunity. 2010;32(6):790-802.

14. Vasquez-Dunddel D, et al. STAT3 regulates arginase-l in myeloid-derived suppressor cells from cancer patients. J Clin Invest. 2013;123(4):1580-9.

15. Bronte $V$, et al. Recommendations for myeloid-derived suppressor cell nomenclature and characterization standards. Nat Commun. 2016;7: 12150.

16. Solito $\mathrm{S}$, et al. A human promyelocytic-like population is responsible for the immune suppression mediated by myeloid-derived suppressor cells. Blood. 2011;118(8):2254-65.

17. Budczies J, et al. Cutoff finder: a comprehensive and straightforward web application enabling rapid biomarker cutoff optimization. PLoS One. 2012; 7(12):e51862.

18. Carstens JL, et al. Spatial computation of intratumoral T cells correlates with survival of patients with pancreatic cancer. Nat Commun. 2017:8:15095.

19. Binnewies $M$, et al. Understanding the tumor immune microenvironment (TIME) for effective therapy. Nat Med. 2018;24(5):541-50.

20. Resovi A, et al. Soluble stroma-related biomarkers of pancreatic cancer EMBO Mol Med. 2018;10:8.
21. Piro $G$, et al. A circulating $\mathrm{TH} 2$ cytokines profile predicts survival in patients with resectable pancreatic adenocarcinoma. Oncoimmunology. 2017;6(9): e1322242.

22. Balachandran VP, et al. Identification of unique neoantigen qualities in longterm survivors of pancreatic cancer. Nature. 2017;551(7681):512-6.

23. Lohneis $P$, et al. Cytotoxic tumour-infiltrating $T$ lymphocytes influence outcome in resected pancreatic ductal adenocarcinoma. Eur J Cancer. 2017; 83:290-301.

24. Fridman $\mathrm{WH}$, et al. The immune contexture in human tumours: impact on clinical outcome. Nat Rev Cancer. 2012;12(4):298-306.

25. Mandruzzato $S$, et al. Toward harmonized phenotyping of human myeloidderived suppressor cells by flow cytometry: results from an interim study. Cancer Immunol Immunother. 2016:65(2):161-9.

26. Ugel $\mathrm{S}$, et al. Immune tolerance to tumor antigens occurs in a specialized environment of the spleen. Cell Rep. 2012;2(3):628-39.

27. Walter $\mathrm{S}$, et al. Multipeptide immune response to cancer vaccine IMA901 after single-dose cyclophosphamide associates with longer patient survival. Nat Med. 2012;18(8):1254-61

28. Hogger P, Sorg C. Soluble CD163 inhibits phorbol ester-induced lymphocyte proliferation. Biochem Biophys Res Commun. 2001;288(4):841-3.

29. Dawar S, et al. Impaired haematopoietic stem cell differentiation and enhanced skewing towards myeloid progenitors in aged caspase-2-deficient mice. Cell Death Dis. 2016;7(12):e2509.

30. Masters SL, et al. NLRP1 inflammasome activation induces pyroptosis of hematopoietic progenitor cells. Immunity. 2012;37(6):1009-23.

31. Biswas SK. Metabolic reprogramming of immune cells in Cancer progression. Immunity. 2015:43(3):435-49.

32. Cassetta $L$, et al. Human tumor-associated macrophage and monocyte transcriptional landscapes reveal Cancer-specific reprogramming, biomarkers, and therapeutic targets. Cancer Cell. 2019;35(4):588-602 e10.

33. Chan $\mathrm{CH}$, et al. Deciphering the transcriptional complex critical for RhoA gene expression and cancer metastasis. Nat Cell Biol. 2010; 12(5):457-67.

34. Cui $L$, et al. IRF-2 is over-expressed in pancreatic cancer and promotes the growth of pancreatic cancer cells. Tumour Biol. 2012;33(1):247-55

35. Yang Q, et al. Minichromosome maintenance 3 promotes hepatocellular carcinoma radioresistance by activating the NF-kappaB pathway. J Exp Clin Cancer Res. 2019:38(1):263.

36. He M, et al. HIF-1alpha downregulates miR-17/20a directly targeting p21 and STAT3: a role in myeloid leukemic cell differentiation. Cell Death Differ. 2013;20(3):408-18.

37. Haghikia A, et al. STAT3 regulation of and by microRNAs in development and disease. JAKSTAT. 2012;1(3):143-50.

38. Solito $\mathrm{S}$, et al. Methods to measure MDSC immune suppressive activity in vitro and in vivo. Curr Protoc Immunol. 2019:124(1):e61.

39. Rodriguez PC, et al. Arginase I-producing myeloid-derived suppressor cells in renal cell carcinoma are a subpopulation of activated granulocytes. Cancer Res. 2009;69(4):1553-60.

40. Gallina $\mathrm{G}$, et al. Tumors induce a subset of inflammatory monocytes with immunosuppressive activity on CD8+ T cells. J Clin Invest. 2006;116(10): 2777-90.

41. Bronte $\mathrm{V}$, et al. IL-4-induced arginase 1 suppresses alloreactive $T$ cells in tumor-bearing mice. J Immunol. 2003;170(1):270-8

42. Marigo I, et al. T cell Cancer therapy requires CD40-CD40L activation of tumor necrosis factor and inducible nitric-oxide-synthase-producing dendritic cells. Cancer Cell. 2016;30(4):651.

43. De Santo $C_{\text {, et }}$ al. Nitroaspirin corrects immune dysfunction in tumorbearing hosts and promotes tumor eradication by cancer vaccination. Proc Natl Acad Sci U S A. 2005;102(11):4185-90.

44. Mondanelli $\mathrm{G}$, et al. A relay pathway between arginine and tryptophan metabolism confers immunosuppressive properties on dendritic cells. mmunity. 2017;46(2):233-44

45. Javeed N, et al. Immunosuppressive CD14(+)HLA-DR (lo/neg) monocytes are elevated in pancreatic cancer and "primed" by tumor-derived exosomes. Oncoimmunology. 2017;6(1):e1252013.

46. Czystowska-Kuzmicz M, et al. Small extracellular vesicles containing arginase-1 suppress T-cell responses and promote tumor growth in ovarian carcinoma. Nat Commun. 2019:10(1):3000.

47. Steggerda SM, et al. Inhibition of arginase by CB-1158 blocks myeloid cellmediated immune suppression in the tumor microenvironment. J Immunother Cancer. 2017;5(1):101. 
48. Molon B, et al. Chemokine nitration prevents intratumoral infiltration of antigen-specific T cells. J Exp Med. 2011;208(10):1949-62.

49. Mondanelli $G$, et al. The immune regulation in cancer by the amino acid metabolizing enzymes ARG and IDO. Curr Opin Pharmacol. 2017:35:30-9

50. Sasso MS, et al. Low dose gemcitabine-loaded lipid nanocapsules target monocytic myeloid-derived suppressor cells and potentiate cancer immunotherapy. Biomaterials. 2016;96:47-62.

51. Bailey P, et al. Genomic analyses identify molecular subtypes of pancreatic cancer. Nature. 2016;531(7592):47-52.

52. Mace TA, et al. IL-6 and PD-L1 antibody blockade combination therapy reduces tumour progression in murine models of pancreatic cancer. Gut. 2018:67(2):320-32

53. Li J, et al. Tumor cell-intrinsic factors underlie heterogeneity of immune cell infiltration and response to immunotherapy. Immunity. 2018;49(1): 178-193 e7.

54. Evans RA, et al. Lack of immunoediting in murine pancreatic cancer reversed with neoantigen. JCI Insight. 2016;1 (14):1-16.

55. Mariathasan S, et al. TGFbeta attenuates tumour response to PD-L1 blockade by contributing to exclusion of T cells. Nature. 2018;554(7693): 544-8.

56. Ugel S, et al. Therapeutic targeting of myeloid-derived suppressor cells. Curr Opin Pharmacol. 2009;9(4):470-81.

57. Welters MJ, et al. Vaccination during myeloid cell depletion by cancer chemotherapy fosters robust T cell responses. Sci Transl Med. 2016; 8(334):334ra52.

58. Cassetta L, Pollard JW. Targeting macrophages: therapeutic approaches in cancer. Nat Rev Drug Discov. 2018;17:887-904.

59. Guilliams M, Mildner A, Yona S. Developmental and functional heterogeneity of monocytes. Immunity. 2018;49(4):595-613.

\section{Publisher's Note}

Springer Nature remains neutral with regard to jurisdictional claims in published maps and institutional affiliations.

Ready to submit your research? Choose BMC and benefit from:

- fast, convenient online submission

- thorough peer review by experienced researchers in your field

- rapid publication on acceptance

- support for research data, including large and complex data types

- gold Open Access which fosters wider collaboration and increased citations

- maximum visibility for your research: over $100 \mathrm{M}$ website views per year

At $\mathrm{BMC}$, research is always in progress.

Learn more biomedcentral.com/submissions 